DOI 10.4171/JEMS/406

Meng Chen · Qi Zhang

\title{
On a question of Demailly-Peternell-Schneider
}

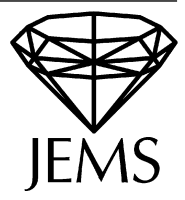

Dedicated to the memory of Eckart Viehweg

Received October 12, 2011 and in revised form February 5, 2012

Abstract. We give an affirmative answer to question posed by Demailly-Peternell-Schneider [DPS] in 2001 and recently by Peternell in [P]. Let $f: X \rightarrow Y$ be a surjective morphism from a $\log$ canonical pair $(X, D)$ onto a $\mathbb{Q}$-Gorenstein variety $Y$. If $-\left(K_{X}+D\right)$ is nef, we show that $-K_{Y}$ is pseudo-effective.

\section{Introduction}

Projective varieties with numerically effective anticanonical bundles appear naturally in the minimal model program. In the surface case, these objects include del Pezzo surfaces, K3 surfaces, Abelian surfaces and so on. The higher dimensional case has been investigated intensively by several authors, for instance, Campana [C], Demailly-PeternellSchneider [DPS], Peternell [P] and the second author [Z2]. A key step in understanding the geometry of these varieties is determining projective morphisms between them. Let $f: X \rightarrow Y$ be a surjective morphism with $-K_{X}$ nef. What can one say about $-K_{Y}$ ? In this direction, one of the main open questions was asked by Demailly-PeternellSchneider [DPS] and recently by Peternell [P]:

Question A. Let $X$ be a complex projective manifold on which $-K_{X}$ is nef. Let $f$ : $X \rightarrow Y$ be a projective morphism onto the projective manifold $Y$. Is $-K_{Y}$ pseudoeffective?

Throughout, we work over the complex number field $\mathbb{C}$.

The main result in this paper is an affirmative answer to Question A. We prove the following stronger theorem:

Main Theorem. Let $X$ be a normal projective variety and $D$ an effective $\mathbb{Q}$-divisor on $X$ such that the pair $(X, D)$ is log canonical. Let $Y$ be a normal and $\mathbb{Q}$-Gorenstein projective variety. If $f: X \rightarrow Y$ is a surjective morphism and $-\left(K_{X}+D\right)$ is nef, then $-K_{Y}$ is pseudo-effective.

M. Chen: Department of Mathematics \& LMNS, Fudan University, Shanghai 200433, China; e-mail: mchen@fudan.edu.cn

Q. Zhang: Department of Mathematics, University of Missouri, Columbia, MO 65211, USA; e-mail: qi@math.missouri.edu

Mathematics Subject Classification (2010): 14E30, 14F10 
Remark 1.1. Naively, under the same condition as in the main theorem, one might expect that $-K_{Y}$ is nef. Unfortunately, this is false in general.

The following example was kindly provided to us by Y. Prokhorov:

Example 1.2. Embed $\mathbb{P}^{1} \times \mathbb{P}^{1}$ into $\mathbb{P}^{5}$ through the linear system $|(1,2)|$ to obtain a projective cone $W \subset \mathbb{P}^{6}$ over the surface $S \cong \mathbb{P}^{1} \times \mathbb{P}^{1}$ with the normal sheaf $\mathcal{N}_{S / W} \cong$ $\mathcal{O}_{S}(-1,-2)$ and vertex $Q=[0,0,0,0,0,0,1]$. Let $\pi: X \rightarrow W$ be the blow-up at the vertex $Q \in W \subset \mathbb{P}^{6}$. Then $X$ is a nonsingular $\mathbb{P}^{1}$-bundle over $S$ with the exceptional divisor $E \cong S$. Since $K_{X}=\pi^{*}\left(K_{W}\right)+E,-K_{X}=-\pi^{*}\left(K_{W}\right)-E$ is nef, one of the curve families on $S$ is $K_{X}$-trivial, while another curve family $\{l\}$ on $S$ is $K_{X}$-negative. Mori's $K$-negative contraction $f=$ Cont $_{l}: X \rightarrow Y$ maps $S$ onto a curve $C=f(S) \cong \mathbb{P}^{1}$ on $Y$. By Mori's theorem $[\mathrm{M}], Y$ is nonsingular. According to Proposition 4.5 and Corollary 4.6 of Prokhorov [Pr], it follows that $K_{Y} \cdot C=2>0$. Thus $-K_{Y}$ is not nef.

In the above example, $f$ is birational. Recently, we learned from Fujino and Gongyo's paper [FG] that $\mathrm{H}$. Sato had constructed the following example where $f: X \rightarrow Y$ is flat:

Example 1.3. Let $\Sigma$ be the fan in $\mathbb{R}^{3}$ whose rays are generated by $x_{1}=(1: 0: 1)$, $x_{2}=(0: 1: 0), x_{3}=(-1: 3: 0), x_{4}=(0:-1: 0), y_{1}=(0: 0: 1)$ and $y_{2}=$ $(0: 0:-1)$. Their maximal cones are $\left\langle x_{1}, x_{2}, y_{1}\right\rangle,\left\langle x_{1}, x_{2}, y_{2}\right\rangle,\left\langle x_{2}, x_{3}, y_{1}\right\rangle,\left\langle x_{2}, x_{3}, y_{2}\right\rangle$, $\left\langle x_{3}, x_{4}, y_{1}\right\rangle,\left\langle x_{3}, x_{4}, y_{2}\right\rangle,\left\langle x_{4}, x_{1}, y_{1}\right\rangle$ and $\left\langle x_{4}, x_{1}, y_{2}\right\rangle$. One can see that $X(\Sigma)$, the toric threefold corresponding to the fan $\Sigma$, has a $\mathbb{P}^{1}$-fibration $f$ onto $Y=\mathbb{P}_{\mathbb{P} 1}\left(\mathcal{O}_{\mathbb{P}^{1}} \oplus \mathcal{O}_{\mathbb{P l}}(3)\right)$ (see [FG] for more details). In fact, $X$ is a weak Fano threefold, but $-K_{Y}$ is not nef.

Remark 1.4. If we replace the condition that $-\left(K_{X}+D\right)$ is nef by $-\left(K_{X}+D\right)$ is pseudo-effective, then the conclusion is again false. This may explain the motivation behind Question A.

The following example is found in [Z1]:

Example 1.5. Let $C$ be a smooth curve of genus $g$ and $A$ an ample line bundle on $C$ such that $\operatorname{deg} A>2 \operatorname{deg} K_{C}$. Let $S=\operatorname{Proj}_{C}\left(\mathcal{O}_{C}(A) \oplus \mathcal{O}_{C}\right)$. Then $K_{S}=\pi^{*}\left(K_{C}+A\right)-2 L$, where $L$ is the tautological bundle. Clearly $-K_{S}$ is big (since $h^{0}\left(S,-m K_{S}\right) \approx c \cdot m^{2}$ for $m \gg 0$ and some constant $c>0$ ). Thus $-K_{S}$ is pseudo-effective. In this setting, $S$ is a $\mathbb{P}^{1}$-bundle over $C$, but $-K_{C}$ is never pseudo-effective if $g>1$. This example suggests the necessity of the condition that $(X, D)$ is log canonical. Indeed, if we let $D=-K_{S}=2\left(L-\pi^{*} A\right)+\pi^{*}\left(A-K_{C}\right)$, the pair $(S, D)$ is not log canonical.

\section{Proof of the Main Theorem}

Before we prove our Main Theorem, let us recall some related definitions and known results. For general references on the minimal model program, see (for instance) [K-M, $\mathrm{KMM}]$. 
Definition 2.1 (Viehweg [V]). Let $X$ be a smooth (quasi-)projective variety and $\mathcal{F}$ a torsion-free coherent sheaf on $X$. We say that $\mathcal{F}$ is weakly positive on $X$ if there exists some Zariski open subvariety $U \subset X$ such that for every ample invertible sheaf $\mathcal{H}$ and every positive integer $m$, there exists some positive integer $n$ such that $\hat{\mathcal{S}}^{m n}(\mathcal{F}) \otimes \mathcal{H}^{n}$ is generated by global sections over $U$, where $\hat{\mathcal{S}}^{n}$ denotes the reflexive hull of $\mathcal{S}^{n}$.

In the proof of our theorem, we need the following weak positivity statement that was originally developed by Viehweg [V] and, later on, generalized by Campana [C] and $\mathrm{Lu}[\mathrm{L}]$.

Lemma 2.2. Let $V$ be a smooth projective variety and $M$ an effective $\mathbb{Q}$-divisor on $V$ such that the pair $(V, M)$ is $\log$ canonical. If $\varphi: V \rightarrow W$ is a surjective morphism onto the nonsingular variety $W$, then $\varphi_{*} m\left(K_{V / W}+M\right)$ is torsion free and weakly positive for every $m \in \mathbb{Z}_{>0}$ such that $m\left(K_{V / W}+M\right)$ is Cartier.

Proof of the Main Theorem. Let $p: Y^{\prime} \rightarrow Y$ be a resolution of $Y$. Let $\pi: X^{\prime} \rightarrow X$ be a $\log$ resolution of $(X, D)$ such that the induced rational map $X^{\prime}-\rightarrow Y^{\prime}$ is in fact a morphism $g^{\prime}: X^{\prime} \rightarrow Y^{\prime}$. We have the following commutative diagram:

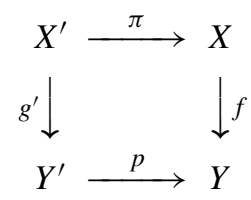

Since the pair $(X, D)$ is log canonical, we have

$$
K_{X^{\prime}}=\pi^{*}\left(K_{X}+D\right)+\sum d_{i} E_{i}
$$

where $d_{i} \geq-1$ for each $i$ and $\sum E_{i}$ is a divisor with normal crossings only. We may also assume that the support of $f^{\prime-1}(\operatorname{Sing}(Y))$ is a divisor with normal crossings only, where $f^{\prime}=f \circ \pi$. Set $Y^{0}:=Y-\operatorname{Sing}(Y)$.

Pick an ample divisor $L$ on $Y$ and a rational number $\delta>0$. Our goal is to show that $-K_{Y}+2 \delta L$ is equivalent to an effective $\mathbb{Q}$-divisor, which implies that $-K_{Y}$ is pseudoeffective.

Let $A$ be a sufficiently very ample divisor on $X$. By Serre's theorem, for every rational number $\varepsilon>0$ and every divisible integer $m>0$ such that $\varepsilon / \delta$ is sufficiently small and $m \delta$ and $m \varepsilon$ are integers, the sheaf

$$
\mathcal{O}_{Y}(m \delta L) \otimes\left(f_{*}^{\prime} \mathcal{O}_{X^{\prime}}\left(m \varepsilon \pi^{*}(A)\right)\right)^{*}
$$

is generated by global sections. Thus, for all integers $n>0$, it follows that $\mathcal{O}_{Y}(n m \delta L) \otimes \bigotimes^{n}\left(f_{*}^{\prime} \mathcal{O}_{X^{\prime}}\left(m \varepsilon \pi^{*}(A)\right)\right)^{*} \quad$ and $\quad \mathcal{O}_{Y}(n m \delta L) \otimes S^{n}\left(\left(f_{*}^{\prime} \mathcal{O}_{X^{\prime}}\left(m \varepsilon \pi^{*}(A)\right)\right)^{*}\right)$ are both generated by global sections. 
Since $-\left(K_{X}+D\right)$ is nef by assumption, $-\left(K_{X}+D\right)+\varepsilon A$ is $\mathbb{Q}$-ample. So we may assume that $-\pi^{*}\left(K_{X}+D\right)+\varepsilon \pi^{*}(A)$ is $\mathbb{Q}$-equivalent to an effective $\mathbb{Q}$-divisor. We may also assume that

$$
\operatorname{Supp}\left(-\pi^{*}\left(K_{X}+D\right)+\varepsilon \pi^{*}(A)+\sum E_{i}\right)
$$

is a divisor with normal crossings only. Let $\Delta=-\pi^{*}\left(K_{X}+D\right)+\varepsilon \pi^{*}(A)+\sum a_{i} E_{i}$, where $a_{i}=-d_{i}$ whenever $d_{i}<0$ and $a_{i}=0$ otherwise.

We may write

$$
K_{X^{\prime} / Y}+\Delta \sim_{\mathbb{Q}} \varepsilon \pi^{*}(A)-f^{\prime *} K_{Y}+\hat{\Delta}
$$

where $\hat{\Delta}:=\sum_{j \in J} d_{j} E_{j}, d_{j} \geq 0$ and $E_{j}$ is $\pi$-exceptional for each $j \in J$. Clearly the pair $\left(X^{\prime}, \Delta\right)$ is $\log$ canonical.

Choose a divisible sufficiently large integer $m>0$ such that $m \varepsilon A, m \delta L, m d_{j} E_{j}$ and $-m K_{Y}$ are all Cartier and define

$$
\omega:=m\left(\varepsilon \pi^{*}(A)-f^{\prime *} K_{Y}+\hat{\Delta}\right) .
$$

By Lemma 2.2, there is a Zariski open smooth subvariety $U \subset Y$ with $\operatorname{codim}(Y-U) \geq 2$ such that

$$
f_{*}^{\prime} \omega=f_{*}^{\prime} \mathcal{O}_{X^{\prime}}\left(m\left(\varepsilon \pi^{*}(A)+\hat{\Delta}\right)\right) \otimes \mathcal{O}_{Y}\left(-m K_{Y}\right)
$$

is locally free and weakly positive over $U$. Recall that $\delta L$ is an ample $\mathbb{Q}$-divisor on $Y$. Since each $E_{j}(j \in J)$ is exceptional with respect to $\pi$, the effect of $\hat{\Delta}$ can be neglected when $f_{*}^{\prime} \omega$ is restricted to $U$. Thus

$$
\left.f_{*}^{\prime} \mathcal{O}_{X^{\prime}}\left(m \varepsilon \pi^{*}(A)\right)\right|_{U}=\left.\left.f_{*}^{\prime} \omega\right|_{U} \otimes \mathcal{O}_{Y}\left(m K_{Y}\right)\right|_{U}
$$

is locally free over $U$. Furthermore, weak positivity implies that

$$
\begin{aligned}
& \left.\left.\left(\mathcal{S}^{n} f_{*}^{\prime} \omega\right)\right|_{U} \otimes \mathcal{O}_{Y}(n m \delta L)\right|_{U} \\
& \quad=\left.\left.\left.\mathcal{S}^{n}\left(f_{*}^{\prime} \mathcal{O}_{X^{\prime}}\left(m \varepsilon \pi^{*}(A)+\sum m d_{j} E_{j}\right)\right)\right|_{U} \otimes \mathcal{O}_{Y}\left(-n m K_{Y}\right)\right|_{U} \otimes \mathcal{O}_{Y}(n m \delta L)\right|_{U} \\
& \quad=\left.\left.\left.\mathcal{S}^{n}\left(f_{*}^{\prime} \mathcal{O}_{X^{\prime}}\left(m \varepsilon \pi^{*}(A)\right)\right)\right|_{U} \otimes \mathcal{O}_{Y}\left(-n m K_{Y}\right)\right|_{U} \otimes \mathcal{O}_{Y}(n m \delta L)\right|_{U}
\end{aligned}
$$

is generated by global sections at the generic point of $U$ for some sufficiently large $n$. Since there is a functorial isomorphism between $S^{n}\left(\mathcal{F}^{*}\right)$ and $\left(S^{n} \mathcal{F}\right)^{*}$ for locally free sheaves, we have a nontrivial trace map

$$
\left.\left.\mathcal{S}^{n}\left(\left(f_{*}^{\prime} \mathcal{O}_{X^{\prime}}\left(m \varepsilon \pi^{*}(A)\right)\right)^{*}\right)\right|_{U} \otimes \mathcal{S}^{n}\left(f_{*}^{\prime} \mathcal{O}_{X^{\prime}}\left(m \varepsilon \pi^{*}(A)\right)\right)\right|_{U} \rightarrow \mathcal{O}_{U}
$$

As we have seen,

$$
\left.\left.\mathcal{O}_{Y}(n m \delta L)\right|_{U} \otimes \mathcal{S}^{n}\left(\left(f_{*}^{\prime} \mathcal{O}_{X^{\prime}}\left(m \varepsilon \pi^{*}(A)\right)\right)^{*}\right)\right|_{U}
$$

is generated by global sections over $U$. Thus

$$
\begin{aligned}
\mathcal{E}_{U}:= & \left.\left.\left.\left.\mathcal{O}_{Y}(n m \delta L)\right|_{U} \otimes \mathcal{S}^{n}\left(\left(f_{*}^{\prime} \mathcal{O}_{X^{\prime}}\left(m \epsilon \pi^{*}(A)\right)\right)^{*}\right)\right|_{U} \otimes\left(\mathcal{S}^{n} f_{*}^{\prime} \omega\right)\right|_{U} \otimes \mathcal{O}_{Y}(n m \delta L)\right|_{U} \\
= & \left.\mathcal{O}_{Y}\left(-n m K_{Y}+2 n m \delta L\right)\right|_{U} \\
& \otimes\left(\left.\left.\mathcal{S}^{n}\left(\left(f_{*}^{\prime} \mathcal{O}_{X^{\prime}}\left(m \varepsilon \pi^{*}(A)\right)\right)^{*}\right)\right|_{U} \otimes \mathcal{S}^{n}\left(f_{*}^{\prime} \mathcal{O}_{X^{\prime}}\left(m \varepsilon \pi^{*}(A)\right)\right)\right|_{U}\right)
\end{aligned}
$$


is generated by global sections at the generic point of $U$. There are induced maps

$$
\left.\bigoplus \mathcal{O}_{U} \stackrel{\alpha}{\rightarrow} \mathcal{E}_{U} \stackrel{\beta}{\rightarrow} \mathcal{O}_{Y}\left(-n m K_{Y}+2 n m \delta L\right)\right|_{U}
$$

such that $\alpha$ is surjective and $\beta$ is nonzero. This proves that

$$
H^{0}\left(U,\left.\mathcal{O}_{Y}\left(-n m K_{Y}+2 n m \delta L\right)\right|_{U}\right) \neq 0 .
$$

Because $\operatorname{codim}(Y-U) \geq 2$, the divisor $n\left(-m K_{Y}+2 m \delta L\right)$ is effective on $Y$.

As instant applications, we can improve some earlier results in [Z1, Z2].

Corollary 2.3. Let $X$ be a normal projective variety and $D$ an effective $\mathbb{Q}$-divisor on $X$ such that the pair $(X, D)$ is log canonical and that $-\left(K_{X}+D\right)$ is nef. Let $f: X \rightarrow Y$ be a surjective morphism onto $Y$ which is a $\mathbb{Q}$-Gorenstein projective variety. Then either

(1) $Y$ is uniruled, or

(2) $K_{Y} \sim_{\mathbb{Q}} 0$.

Proof. If $Y$ is not uniruled, then $K_{Y}$ is pseudo-effective by [BDPP]. However our Main Theorem says $-K_{Y}$ is pseudo-effective, which means $K_{Y} \sim_{\mathbb{Q}} 0$.

Remark 2.4. The weaker statement corresponding to (2) in [Z2] is that the Kodaira dimension $\kappa(Y)$ is zero.

Corollary 2.5 (cf. [Z1]). Let $X$ be a smooth projective variety with $-K_{X}$ nef. Then the Albanese map $\operatorname{Alb}_{X}: X \rightarrow \operatorname{Alb}(X)$ is surjective and has connected fibers.

Proof. Let $a: X \rightarrow V$ be the induced morphism with connected fibers after the Stein factorization of $\operatorname{Alb}_{X}$. We may even assume that $V$ is smooth. Then $\kappa(V)=0$ by the Main Theorem, and Kawamata's theorem $[\mathrm{K}]$ implies that $V$ is an abelian variety. Thus it follows that $V=\operatorname{Alb}(X)$ due to the universal property of $\operatorname{Alb}_{X}$.

Acknowledgments. Chen thanks the Max-Planck-Institut für Mathematik (Bonn) for the invitation and the generous support in 2011. Zhang thanks the Department of Mathematics and the Key Laboratory of Mathematics for Nonlinear Sciences (MOE), Fudan University for the hospitality and support during his visit in the summer of 2011 when part of the paper was written.

The first author was supported by National Natural Science Foundation of China (\# 11171068), NSFC for Innovative Research Groups (\# 11121101) and Doctoral Fund of Ministry of Education of China (\# 20110071110003).

\section{References}

[BDPP] Boucksom, S., Demailly, J., Păun, M., Peternell, T.: The pseudo-effective cone of a compact Kähler manifolds and varieties of negative Kodaira dimension. J. Algebraic Geom. 22, 201-248 (2013) Zbl pre06154993 MR 3019449

[C] Campana, F.: Orbifolds, special varieties and classification theory. Ann. Inst. Fourier (Grenoble) 54, 499-630 (2004) Zbl 1062.14014 MR 2097416 
[DPS] Demailly, J., Peternell, T., Schneider, M.: Pseudo-effective line bundles on compact Kähler manifolds. Int. J. Math. 12, 689-741 (2001) Zbl 1111.32302 MR 1875649

[FG] Fujino, O., Gongyo, Y.: On images of weak Fano manifolds. Math. Z. 270, 531-544 (2011) Zbl 1234.14033 MR 2875847

[K] Kawamata, Y.: Characterization of abelian varieties. Compos. Math. 43, 253-276 (1981) Zbl 0471.14022 MR 0622451

[KMM] Kawamata, Y., Matsuda, K., Matsuki, K.: Introduction to the minimal model problem. Adv. Stud. Pure Math. 10, 283-360 (1987) Zbl 0672.14006 MR 0946243

[K-M] Kollár, J., Mori, S.: Birational Geometry of Algebraic Varieties. Cambridge Univ. Press (1998) Zbl 0926.14003 MR 1658959

[L] Lu, S.: A refined Kodaira dimension and its canonical fibration. arXiv:math/0211029

[M] Mori, S.: Threefolds whose canonical bundles are not numerically effective. Ann. of Math. 116, 133-176 (1982) Zbl 0557.14021 MR 0662120

[P] Peternell, T.: Varieties with generically nef tangent bundles. J. Eur. Math. Soc. 14, 571603 (2012) Zbl 1250.14022 MR 2881306

[Pr] Prokhorov, Y.: On the degree of Fano threefolds with canonical Gorenstein singularities. Russian Acad. Sci. Sb. Math. 196, 81-122 (2005) Zbl 1081.14058 MR 2141325

[V] Viehweg, E.: Weak positivity and the additivity of Kodaira dimension for certain fiber spaces. Adv. Stud. Pure Math. 1, 329-353 (1983) Zbl 0513.14019 MR 0715656

[Z1] Zhang, Q.: On projective manifolds with nef anticanonical bundles. J. Reine Angew. Math. 478, 57-60 (1996) Zbl 0855.14007 MR 1409052

[Z2] Zhang, Q.: On projective varieties with nef anticanonical divisors. Math. Ann. 332, 697703 (2005) Zbl 1083.14010 MR 2181470 